\title{
INVERSIVE SEMIRINGS
}

PAUL H. KARVELLAS

(Received 7 September 1972)

Communicated by G. B. Preston

\section{Introduction}

A semiring $(S,+, \cdot)$ is a nonempty set $S$, endowed with associative operations of addition and multiplication, such that the multiplicative semigroup $(S, \cdot)$ distributes over the addition. That is: $x(y+z)=x y+x z$ and $(x+y) z=x z+y z$ for all $x, y$ and $z$ in $S$. A topological semiring is a semiring, defined on a Hausdorff space, such that both of the operations are jointly continuous.

In this note we consider the extension to semirings of the concept of an inverse semigroup [2, pp. 26-34]. The behaviour of additive subgroups is examined first. Several sufficient conditions are given for an additively inversive semiring to be a union of additive groups. A characterization of an additively inversive, multiplicatively idempotent semiring is given as a union of Boolean subrings with commuting additive identities. We conclude with the specification of all doubly inversive topological semirings defined on a continuum irreducible between two points (i.e. a topological arc).

Ideals, idempotents and subgroups are defined for each semigroup structure separately. The subset $A$ is a multiplicative (additive) ideal of the semiring $S$ if for each $a$ in $A$ and $s$ in $S$, both $a s$ and $s a(a+s, s+a)$ are contained in $A$. The two idempotent sets are $E[+]=\{x: x=x+x\}$ and $E[\cdot]=\left\{x: x=x^{2}\right\}$. Kernels, or minimal ideals, if such exist, are necessarily unique and will be written $K[+]$ and $K[\cdot]$. The maximal additive subgroup $H[+](e)$ associated with an additive idempotent $e$ is the set $(e+S+e) \cap\{x: e \in(x+S) \cap(S+x)\}$. The union of all additive groups will be denoted by $H[+]$. Similarly we have $H[\cdot](k)$, for $k \in E[\cdot]$, and the union $H[\cdot]$ of all multiplicative subgroups. A semi-ideal is a multiplicative ideal which is closed under addition. For $n$ a positive integer and $x$ an element of the semiring, $n x$ is the $n$-fold sum of $x$ : if the semiring has a multiplicative identity, interpret $n x$ as the product of two elements of the semiring under multiplication.

A semiring is additively (multiplicatively) regular if for each element $x$ 
in $S$ there exists an element $x^{\prime}\left(x^{*}\right)$ such that $x=x+x^{\prime}+x\left(x=x \cdot x^{*} \cdot x\right)$. If, in addition, the element $x^{\prime}\left(x^{*}\right)$ is unique, and satisfies $x^{\prime}=x^{\prime}+x+x^{\prime}\left(x^{*}\right.$ $\left(x^{*}=x^{*} \cdot x \cdot x^{*}\right)$, then $S$ is an additively (multiplicatively) inversive semiring. Equivalently for the additive semigroup [2], $(S,+)$ is inversive if and only if $(S,+)$ is regular and any two additive idempotents commute with one another under addition. A similar statement applies to the multiplicative semigroup. Finally, $S$ is a doubly inversive semiring if both $(S,+)$ and $(S, \cdot)$ are inverse semigroups.

Trivially $H[+]$ is nonempty if and only if $E[+]$ is nonvoid. If $S$ is a compact topological semiring, kernels and idempotent sets exist [10]. For a fixed element $s \in S$, the maps $x \rightarrow s x$ and $x \rightarrow x s$ are additive homomorphisms, thereby preserving both additive idempotents and additive groups. Consequently $E[+]$ and $H[+]$ are multiplicative ideals. In general neither set need be closed under addition, but for commutative addition in $H[+]$ both sets are semi-ideals. If $t$ is a right multiplicative identity for $E[+]$, then $E[+]$ is a semi-ideal since

$$
\begin{aligned}
(e+f)+(e+f) & =(e+f)(t+t) \\
& =e(t+t)+f(t+t)=e+f
\end{aligned}
$$

for any two elements $e$ and $f$ in $E[+]$. The example below, with all products defined to be the element $a$, illustrates that even such conditions as finiteness of the semiring need not force additive closure on $H[+]$ and $E[+]$.

\begin{tabular}{l|lllll} 
\pm & $a$ & $b$ & $c$ & $d$ & $e$ \\
\hline$a$ & $a$ & $d$ & $a$ & $d$ & $e$ \\
$b$ & $e$ & $b$ & $c$ & $e$ & $e$ \\
$c$ & $c$ & $b$ & $c$ & $b$ & $e$ \\
$d$ & $e$ & $d$ & $a$ & $e$ & $e$ \\
$e$ & $e$ & $e$ & $e$ & $e$ & $e$
\end{tabular}

The first result concerns commutativity of addition in the additive subgroups of a semiring when certain conditions have been imposed on the multiplication.

THEOREM 1. Let $(S,+, \cdot)$ be a semiring.

(1) If $a \in a S$ for each $a$ in $S$, then each additive subgroup of $S$ is commutative.

(2) If $S=E[\cdot]$, then $E[+]=\{x+x: x \in S\}$ and every maximal additive subgroup is a Boolean ring.

Proof. Recall [2] that every additive subgroup is contained in a maximal additive subgroup and distinct maximal subgroups are disjoint. If $E[+]$ is empty 
the conclusion of (1) holds vacuously. Let $a$ and $b$ be elements of $H[+](e)$ for some $e$ in $E[+]$. There exist $s$ and $t$ in $S$ such that $a=a s$ and $b=b t$. Since $a=a s \in H[+](e) . s \subset H[+](e s)$ and $b \in H[+](e) . t \subset H[+](e t)$, requiring that $e=e s=e t$, the maps $x \rightarrow x s$ and $x \rightarrow x t$ carry $H[+](e)$ into itself. Both $a t$ and $b s$ are thus in $H[+](e)$ and therefore

$$
\begin{aligned}
(a+b)(t+s) & =a t+a+b+b s \\
& =a t+b+a+b s .
\end{aligned}
$$

Cancelling in $H[+](e)$ we obtain the result $a+b=b+a$.

Assume now that $S=E[\cdot]$. For $x$ in $S, x+x=(x+x)^{2}=4 x^{2}=2 x+2 x \in$ $E[+]$. The reverse inclusion is trivial. Thus $E[+]=\{x+x: x \in S\}$ and is nonempty. From a result in [4], a maximal additive subgroup $H[+](e)$ is closed under multiplication if and only if $e \in E[\cdot]$. From part (1) and $S=E[\cdot]$, each additive subgroup is commutative. Each maximal subgroup is thus a subring, contained in $E[\cdot]$, and must be a Boolean ring.

Clearly the existence of a one-sided multiplicative identity implies commutativity of addition in any additive subgroup. And, if $a \in a S \cup S a$ for each $a$ in $S$, every additive subgroup is then the union of commutative subgroups which need not be those generated by a single element. For example, let $I$ be $[0,1]$ under max multiplication and min addition, and $R$ be a Boolean ring of order $>3$. Then the semiring $S=R \times I$, under coordinate-wise operations, is the union of the additively commutative subgroups $R \times\{y\}(y \in I)$.

THEOREM 2. Let $(S,+, \cdot)$ be a semiring which is the union of additive groups. Then $S$ is the union of all maximal additive groups and the product of any two additive groups under multiplication is contained wholly within a third.

Proof. The first conclusion follows from a result in [2]. Let $e, f \in E[+]$. We show that $H[+](e) . H[+](f) \subset H[+](e f)$. Similarly if $G$ and $G^{\prime}$ are additive subgroups contained in $H[+](e)$ and $H[+](f)$ respectively, $G . G^{\prime} \subset$ $H[+](e f)$. Let $-x$ denote the additive inverse of the element $x$ of $H[+](e)$. Then $e . H[+](f) \subset H[+](e f) \cap E[+]=\{e f\}$. For $y \in H[+](f)$, we have that $e y=e f$ and also that

$$
\begin{aligned}
& x y+e y=(x+e) y=x y=(e+x) y=e y+x y \\
& x y+(-x) y=(x-x) y=e y=(-x) y+x y
\end{aligned}
$$

Therefore $x y \in(e f+S+e f) \cap\{s: e f \in(s+S) \cap(S+s)\}=H[+](e f)$. 


\section{Regular and Inversive Semirings}

The following results are well known $[2 ; 6]$. In essence the additive inverse, denoted by ('), behaves in a manner closely resembling the behaviour of the minus sign in ordinary arithmetic.

THEOREM 3. Let $(S,+, \cdot)$ be an additively inverse semiring.

(1) $E[+]$ is additively commutative and is a semi-ideal.

(2) For $x$ and $y$ in $S, x=\left(x^{\prime}\right)^{\prime},(x+y)^{\prime}=y^{\prime}+x^{\prime},(x y)^{\prime}=x^{\prime} \cdot y=x \cdot y^{\prime}$ and $x, y=x^{\prime}, y^{\prime}$.

(3) $S$ is a union of additive groups if and only if for each $x$ in $S$ the additive idempotents $x+x^{\prime}$ and $x^{\prime}+x$ are equal.

THEOREM 4. Let $(S,+, \cdot)$ be an additively inversive semiring with a right identity $t$ for multiplication.

(1) $(S,+)$ is commutative and $S=H[+]$.

(2) If $e=t+t^{\prime}$, then $e \in E[+] \cap E[\cdot]$ and $H[+](e)$ is a subring with right identity $t$.

(3) If $S$ has a minimal additive ideal $K[+]$, then $K[+]$ is a subring.

Proof. To obtain $S=H[+]$ we need only show that addition in $S$ is commutative. For $x, y \in S$ we obtain the equations

$$
\begin{aligned}
x+y=(x+y) t & =(x+y)^{\prime} \cdot\left(t^{\prime}\right) \\
& =\left(y^{\prime}+x^{\prime}\right) \cdot t^{\prime} \\
& =y t+x t=y+x .
\end{aligned}
$$

Therefore addition is commutative. Since $t=t+t^{\prime}+t$, the element $e=t+t^{\prime}$ is in $E[+]$. But $t=t^{2}$, implying that $t^{\prime}=t \cdot t^{\prime}$ and therefore we obtain the result

$$
e^{2}=t^{2}+t^{\prime} t+t t^{\prime}+t^{\prime} t^{\prime}=t+t^{\prime}+t^{\prime}+t=e .
$$

Consequently $e \in E[\cdot]$ and $H[+](e)$ is a multiplicative subsemigroup [4]. Because addition is commutative, $H[+](e)$ is thus a subring and the right identity $t$ is easily shown to be an element of $H[+](e)$.

Assume now that $S$ has a minimal additive ideal $K[+]$. Then $K[+]$ is an additive group from the commutativity of addition. Since $S=S^{2}=$ $\{x y: x, y \in S\}, K[+] \cap S^{2}$ is nonempty, implying [8] that $K[+]$ is a multiplicative subsemigroup and therefore is a subring of $S$.

As the next result demonstrates, the commutativity of additon in an additively inversive semiring is not entirely dependent upon the existence of one-sided identities for the multiplication.

THEOREM 5 Let a be an element of an additively inversive semiring $(S,+, \cdot)$. 
Then $a S$ and $S a$ are additively commutative subsemirings of $S$ contained in $H[+]$. If $S=S^{2}$, then $S=H[+]$.

Proof. Let $a \in S$. Since $a x+a y=a(x+y)$ and $a x . a y 1=a(x a y), a S$ is a subsemiring. Similarly $S a$ is a subsemiring. Moreover, we have that

$$
\begin{aligned}
a x+a y=a(x+y) & =a^{\prime} \cdot(x+y)^{\prime} \\
& =a^{\prime} \cdot\left(y^{\prime}+x^{\prime}\right)=a y+a x
\end{aligned}
$$

implying that $(a S,+)$ is commutative. Since $(a x)^{\prime}=a \cdot x^{\prime}, a S$ contains the additive inverse of each of its elements and is an inverse subsemigroup of $(S,+)$. By (3) of Theorem 3, aS is contained in $H[+]$. Similar arguments hold for $S a$ and thus $S^{2}=\cup\{a S: a \in S\} \subset H[+]$. The last conclusion is now obvious.

The set $S^{2}$ need not be an additive subsemigroup, as for example, the set of polynomials in the indeterminate $x$ with positive integer coefficients. Both 4 and $x^{2}$ are in $S^{2}$, but $\left(4+x^{2}\right)$ is not.

THEOREM 6. The following statements are equivalent for the semiring $(S,+$,$) .$

(1) $S=E[\cdot]$ and $(S,+)$ is an inverse semigroup.

(2) $E[+]$ is additively commutative and $S$ is the union of Boolean subrings.

Proof. $1 \rightarrow 2$ : Let $S=E[\cdot]$ and $(S,+)$ be an inverse semigroup. For each element $x$ in $S, x=x \cdot x=x^{\prime} \cdot x^{\prime}=x^{\prime}$. Therefore any two elements of $S$ commute under addition since $a+b=(a+b)^{\prime}=b^{\prime}+a^{\prime}=b+a$. Because addition is commutative and inversive, $S=H[+]$. From Theorem 1 each maximal additive is a Boolean ring.

$2 \rightarrow 1$ : If $S$ is a union of Boolean subrings, then $S=E[\cdot]$ and $(S,+)$ is regular. Because $(E[+],+)$ is a commutative subsemigroup, addition is inversive from a result in [2].

Although multiplication in a Boolean ring is commutative, a semiring of the type considered in Theorem 6 need not have this property. The following example is from [3]. Let $S$ be the set $\{0, a, b, e, e+a, e+b, a+b, e+a+b\}$ with commutative addition, additive identity 0 , multiplicative identity $e$, where $2 e=e$, $a=a^{2}=a b, b=b^{2}=b a$. Then $S$ is an additively inversive semiring, $S=E[\cdot]$ and the maximal additive subgroups are singleton sets.

The next result has an obvious application to additively inversive semirings which are multiplicatively regular.

THEOREM 7. Let $S$ be an additively inversive semiring such that for each element a of $S, a \in a S \cap S a$. Then $S$ is additively commutative and the union of additive groups. 
Proof. We need only demonstrate the commutativity of addition. For elements $x, y \in S$ there exist $m$ and $n$ in $S$ such that $x+y=(x+y) n$ and $y+x=m(y+x)$. Recall from Theorem 5 that $S n$ and $m S$ are additively commutative. Thus we obtain

$$
\begin{aligned}
y+x & =m(y+x)=m(x+y)=m[(x+y) n] \\
& =m[(y+x) n]=[m(y+x)] n=(y+x) n=x+y .
\end{aligned}
$$

COROllary 8. A doubly inversive semiring is additively commutative and is the union of additive groups.

The additive commutativity of Theorem 7 is not obtained if the addition is only regular. The following example has both $(S,+)$ and $(S, \cdot)$ regular, with $S=E[+]=E[\cdot]=H[+]=H[\cdot]=S^{2}$, but neither addition nor multiplication is commutative.

\begin{tabular}{c|ccccc:ccc}
+ & 0 & $a$ & $b$ & & - & 0 & $a$ & $b$ \\
\hline 0 & 0 & $a$ & $b$ & & 0 & 0 & 0 & $b$ \\
$a$ & 0 & $a$ & $b$ & & $a$ & 0 & $a$ & $b$ \\
$b$ & 0 & $a$ & $b$ & & $b$ & 0 & $b$ & $b$
\end{tabular}

Similarly the unit circle, with complex multiplication, can be the multiplicative semigroup of a topological semiring only if the addition is left- or righttrivial [7]. Indeed, if $S$ is a topological semiring in which $S$ is a multiplicative group, with identity element 1 , the addition is regular but cannot be inversive except if $S=\{1\}$. In doubly inversive semirings we have an extension of Theorem 3. Recall that inverses are denoted by (') and (*) for addition and multiplication, respectively.

THEOREM 9. Let $(S,+, \cdot)$ be a doubly inversive semiring. Then for each $x$ in $S,\left(x^{\prime}\right)^{*}=\left(x^{\prime *}\right)^{\prime}$.

Proof. In an additively inversive semiring $(a b)^{\prime}=a \cdot b^{\prime}$ and $a \cdot b=a^{\prime} \cdot b^{\prime}$. Hence $(a b c)^{\prime}=a^{\prime} . b^{\prime} . c^{\prime}$. Let $x$ be in $S$. There exist unique elements $x^{*}$ and $\left(x^{\prime}\right)^{*}$ such that equations (1) and (2) hold.

$$
\begin{aligned}
x^{\prime} & =x^{\prime} \cdot\left(x^{\prime}\right)^{*} \cdot x^{\prime} & \left(x^{\prime}\right)^{*} & =\left(x^{\prime}\right)^{*} \cdot x^{\prime} \cdot\left(x^{\prime}\right)^{*} \\
x & =x \cdot x^{*} \cdot x & x^{*} & =x^{*} \cdot x \cdot x^{*}
\end{aligned}
$$

Taking the additive inverse of the equations of (2) we have

$$
x^{\prime}=x^{\prime} \cdot\left(x^{*}\right)^{\prime} \cdot x^{\prime} \quad\left(x^{*}\right)^{\prime}=\left(x^{*}\right)^{\prime} \cdot x^{\prime} \cdot\left(x^{*}\right)^{\prime} .
$$

Comparing equations (1) and $(3),\left(x^{*}\right)^{\prime}=\left(x^{\prime}\right)^{*}$. 
For doubly inversive topological semirings it is possible to obtain somewhat stronger conclusions.

THEOREM 10. Let $(S,+, \cdot)$ be a compact, doubly inversive topological semiring.

(1) $(S,+)$ is commutative and $S$ is the union of totally disconnected additive groups.

(2) The additive kernel $K[+]$ is a topological ring: if $S$ is connected, then $K[+]$ is a single element in $E[+] \cap E[\cdot]$.

(3) S has a multiplicative zero element.

Proof. The commutativity of addition and $S=H[+]$ follow directly from Corollary 8 . Since $S=E[\cdot] S=S E[\cdot]$ and is also compact, each additive subgroup is totally disconnected [9]. From $S=S^{2}, K[+]$ is a multiplicative subsemigroup [8]. The map $x \rightarrow x^{\prime}$ is continuous [10] and $K[+]$ exists, both from the fact that $S$ is compact. Therefore $K[+]$ is a topological subring of $S$. If $S$ is connected, then $K[+]$ is both connected [10] and also totally disconnected, implying the last part of (2).

The multiplicative kernel $K[\cdot]$ is a group [2] and for any two elements $x$ and $y$ in $K[\cdot]$, we have that

$$
x+y=x\left(x^{*} x\right)+\left(x x^{*}\right) y=x\left(x^{*} x+x^{*} y\right) \in K[\cdot] .
$$

Thus $K[\cdot]$ is a compact, additively commutative semiring which is a multiplicative group. From a result in $[7], K[\cdot]$ must be a single point.

The next result is of general interest. Theorem 11 can also be obtained more directly from the main result in [9].

THEOREM 11. There exists no doubly inversive topological semiring on an indecomposable continuum.

Proof. Let $S$ be a doubly inversive topological semiring defined on an indecomposable continuum. The additive semigroup $(S,+)$ is a group [5] and addition in $S$ is commutative. Hence $\left(S,+,^{\cdot}\right)$ is a topological ring from the fact that the inversion map is continuous. However, the only possible multiplication on a compact and connected topological ring is $x y=0[1]$ and the multiplication is therefore not inversive unless $S$ is a single point.

We conclude this section with an example of a doubly inversive semiring without a multiplicative identity. Since $a d=c \neq b=d a, S$ is not the union of multiplicative groups. 


\begin{tabular}{|c|c|c|c|c|c|c|c|c|c|c|c|}
\hline & 0 & $a$ & $b$ & $c$ & $d$ & & 0 & $a$ & $b$ & $c$ & $d$ \\
\hline$\overline{0}$ & 0 & 0 & $\overline{0}$ & 0 & $\overline{0}$ & 0 & 0 & 0 & 0 & 0 & 0 \\
\hline$a$ & 0 & $a$ & 0 & 0 & 0 & $a$ & 0 & 0 & $a$ & 0 & $c$ \\
\hline$b$ & 0 & 0 & $b$ & 0 & 0 & $b$ & 0 & 0 & $b$ & 0 & $d$ \\
\hline 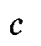 & 0 & 0 & 0 & $c$ & 0 & $c$ & 0 & $a$ & 0 & $c$ & 0 \\
\hline & 0 & 0 & 0 & 0 & $d$ & $d$ & 0 & $b$ & 0 & $d$ & \\
\hline
\end{tabular}

\section{Doubly Inversive Semirings on Topological Arcs}

A topological arc is a continuum irreducibly connected between two points. In the remainder of this section $S$ shall denote a doubly inversive topological semiring $(S,+, \cdot)$ defined on the topological arc $[d, u]$, with $d$ minimal and $u$ maximal in the cutpoint order. Koch and Wallace [5] have characterized all inversive topological semigroups defined on a topological arc. The material presented here is an extension of their results. We shall need the next theorem.

THEOREM 12. Let $S$ be a doubly inversive topological semiring defined on the topological arc $[d, u]$.

(1) Addition and multiplication are commutative and $S=H[+]=H[\cdot]$. If $x \in S \backslash E[+]$, the maximal additive subgroup containing $x$ is the set $\{x, 2 x\}$, where $2 x \in E[+]$. Similarly, if $x \in S \backslash E[\cdot], H[\cdot]\left(x^{2}\right)=\left\{x, x^{2}\right\}$.

(2) The inversion maps $x \rightarrow x^{\prime}$ and $x \rightarrow x^{*}$ are continuous.

(3) $E[+]$ is a connected semi-ideal of $S$ and there exist $e$ and $f$ in $S$, with $e=e \wedge f$, such that $E[+]=[e, f]$.

(4) $E[\cdot]$ is a connected multiplicative subsemigroup and there exist $s$ and $t$ in $S$ such that $E[\cdot]=[s, t]$.

(5) There exist elements $z$ and $m$ in $E[+] \cap E[\cdot]$ such that $K[+]=\{z\}$ and $K[\cdot]=\{m\}$.

(6) Either $e=d$ or $f=u$. Similarly $s=d$ or $t=u$.

Proof. Both (1) and (6) follow from the characterization given in [5]. The inversion maps are continuous since the set $S$ is compact. Because the maps $x \rightarrow\left(x+x^{\prime}\right)$ and $x \rightarrow x \cdot x^{*}$ are retracts onto $E[+]$ and $E[\cdot]$ respectively, the idempotent sets are subcontinua. Both are closed sets in a totally ordered space and elements $e, f, s$ and $t$ exist with the properties as specified. Because both sets, under their respective operations, are commutative, they are subsemigroups. The minimal ideals for addition and multiplication are singleton sets from Theorem 10 .

The following are the three main cases, as stated for the inverse semigroup $(S, \cdot)$. Similar explanation applies to the semigroup $(S,+)$. We assume throughout that $t=u$, from which $E[\cdot]=[s, u]$. 
CASE I. $m=d$, requiring that $s=d$. Therefore $S=E[\cdot]$ and $(S, \cdot)$ is a min thread.

CASE II. $m \neq d, s \neq d$. Then $s=m$ and $E[\cdot]=[m, u]$ is a min thread under multiplication. Let $A$ be the subinterval $[d, m]$. For $x \in A, x^{2}=e_{x} \in E[\cdot]$ and the injective map $x \rightarrow x^{2}$ takes $A$ onto the interval $\left[m, e_{\mathrm{d}}\right] \subset E[\cdot]$. The multiplication is given by:

$$
\begin{aligned}
x \cdot y & =x \wedge y & & \text { for } x, y \in E[\cdot] \\
& =x & & \text { for } x \in A, y \in\left[e_{x}, u\right] \\
& =t & & \text { for } x \in A, y=e_{t} \in\left[m, e_{x}\right] \\
& =e_{x} \wedge e_{y} & & \text { for } x, y \in A .
\end{aligned}
$$

CASE III. $s=d, m \neq d$ and thus $S=E[\cdot]$. Multiplication in $A=[d, m]$ is max, while $B=[m, u]$ is a min thread. The product $A B$ is contained in either $A$ or $B$ and is the subinterval $[d u, m]$ (resp. $[m, d u]$ ).

With the convention $E[\cdot]=[s, u]$, recall that in $E[+]=[e, f]$, at least one of $e$ and $f$ is an endpoint. We obtain the five subcases below.

A. $f=u, z=d$ : hence $e=d, S=E[+]$ and $(S,+)$ is a min thread.

B. $f=u, z \neq d \neq e$ : thus $e=z, E[+]=[z, u]$ and the addition in $E[+]$ is a min thread.

C. $f=u, e=d \neq z$ : therefore $S=E[+]$ and $[d, z]$ and $[z, u]$ are, respectively, additive max and min threads.

D. $e=d, z=u$ : hence $f=u, S=E[+]$ with $\max$ addition.

E. $e=d, z \neq u \neq f$ : thus $z=f$ and addition in the subinterval $[d, z]=E[+]$ is $\max$.

Of the fifteen possible combinations, only seven lead to distinct doubly inverse topological semirings.

CASE I. All semirings with case I multiplication have $x y=x \wedge y$. For subcase $A$ the addition is also min and is the first example of a doubly inversive semiring. Subcase B cannot occur since the element $m(=d)$ is contained in $E[+]=[z, u]$ and $z \neq d$ by assumption.

In subcase $C$ let $A=[d, z]$ and $B=[z, u]$. Note that $A=\{x: x z=x\}$ and $B=\{x: x z=z\}$, while $S=E[+]$. For $a \in A$ and $b \in B,(a+b) z=$ $a z+b z=a+z=z$. It is now easily shown that $A+B=[z, d+u] \subset B$ By considering the function $f(x)=x+u$, we obtain the following characterization. Let $F: S \rightarrow B$ be any continuous function which is the identity on $B$ and reverses order on $A(x y=x$ in $A$ implies $F(x) F(y)=F(y)$ in $B)$. Addition is given by

$$
\begin{aligned}
x+y & =F(x) \cdot y & & \text { for } x=x \wedge y \\
& =x \cdot F(y) & & \text { for } y=x \wedge y
\end{aligned}
$$


Subcase $\mathrm{D}$ is defined by $x+y=x \vee y$ and $x y=x \wedge y$. In subcase $\mathrm{E}$, $S=E[\cdot]$ and $E[+]=[d, z]=\{x+x: x \in S\}$ from Theorem 1. If $x \in[z, u]$, then $z=x z$ and $x+x \in E[+]$. Then $x+x=z(x+x)=2 z x=2 z=z$. Since $x=x^{\prime}$, we have that $x=x+x^{\prime}+x=x+2 x=x+z=z$. Hence $u=z$, which is a contradiction, implying that this subcase cannot occur.

CASE II. All case II examples have $E[\cdot]=[m, u]$, with min multiplication. In subcase $A, z=d$ and is contained in $E[\cdot]$. But $m \neq d$ by assumption and therefore this subcase cannot occur. In subcase $\mathbf{B}, m \neq d$ and $m \in E[+]$, while $z \in E[\cdot]$. Consequently $z=m$. However, if $x \in[d, m]$, then $x^{2} \in E[\cdot]=$ $E[+]$ and $x=x \cdot x^{2} \in x \cdot E[+] \subset E[+]$. That is, $d \in E[+]$, which is a contradiction.

In subcase $\mathrm{C}$ note that $S=E[+]$ and $E[\cdot]=[m, u]$, with $m \neq d$. Since $z \in E[\cdot]$, denote the resulting subintervals by $A=[d, m], B=[m, z]$ and $C=[z, u]$. Both $(A,+)$ and $(B,+)$ are max threads, while $(C,+)$ and $(C, \cdot)$ and $(B, \cdot)$ are min threads. For $a \in A$ and $b \in B, a b=a b+m=m$, from which $A B=\{m\}$. If $a \in A \backslash\{m\}$, the element $a^{2}$ is contained in $E[\cdot]=B \cup C$. If $a^{2} \in B$, then $a=a \cdot a^{2} \in A B=\{m\}$. Hence $a^{2} \in C$ and thus $z=a^{2} z=a(a z)$ $=a m=m$, implying that $z=m$. Relabel the subintervals as $A=[d, m]$, $B=\left[m, e_{d}\right] \subset E[\cdot]$, where $e_{d}=d^{2}$. The injective map $x \rightarrow x^{2}$ takes $A$ onto $B$. If $y=x+y$ in $A$ then $y^{2}=x^{2} y^{2}$ in $B$. Again $(A,+)$ is a max thread, while $C=\left[e_{d}, u\right]$ and $B$ are min threads under both operations. We now show that $A+E[\cdot]=\{m\}$. For $a \in A$ let $p=a+u$. If $p \in A$, then from $a=a u$, $p^{2}=a+a^{2} \in E[\cdot]$. Moreover, $p=p^{3}=\left(a+a^{2}\right)(a+u)=a^{2}+a^{3}+a u+$ $a^{2} u=a+a^{2}=p^{2}$. Consequently $A+u \subset E[\cdot]$. For any $a \in A, a=a+d$ and $a^{2}=a^{2}+a d$, from which $a=a d^{2}=a^{2} d$ and $a^{2}=a d$. Since $(a+u)(d+u)$ $=a+a^{2}=a+u$, then $A+u=[m, d+u] \subset E[\cdot]$. The injective map $x \rightarrow x d$ from $B$ into $A$ maps the element $a^{2}$ onto $a$ for each $a$ in $A$. Since $a+u=a+a^{2} \in B$, for $a \in A$, we obtain finally that $\left(a+a^{2}\right) d=a d+a^{2} d a^{2}$ $+a \in A \cap B=\{m\}$. Therefore $A+u=\{m\}$ and consequently $A+E[\cdot]=\{m\}$ also.

Subcase D cannot occur. For $a \in[d, m]$ and $b \in[m, u], a b+m=a b=m$. Since $a^{2} \in E[\cdot]=[m, u], a=a^{3}=m$. Thus the element $d$ is in $E[\cdot]$, which is a contradiction.

To complete the characterization of case II we show that subcase $\mathrm{E}$ cannot occur. Since $z \in E[\cdot]=[m, u]$, we obtain the subintervals $A=[d, m], B=[m, z]$ $C=[z, u]$, where $E[+]=[d, z]$ is an additive max thread. It is easily shown that $A B=\{m\}$. If $a \in A \backslash\{m\}$ and $a^{2} \in B$, then $a=a^{3}=m$, which is a contradiction. Thus $a^{2} \in C$ and $z=a^{2} z=m$. Let $x \in E[+]$. Then $x^{2} \in E[+] \cap E[\cdot]$ $=\{m\}$, implying that $x=m$. Consequently $d=m$, which contradicts the original hypotheses. 
CASE III: In this set of examples $m \neq d$ and $S=E[\cdot]$. The subintervals $[d, m]$ and $[m, u]$ are, respectively, max and min threads under multiplication. In subcase $A$ the addition is max, with $[d, m] .[m, u]=\{m\}$.

The addition of subcase $B$ will be the additive analogue of the case II inversive multiplication. We outline the method emloyed. Firstly $z=m$, from which the subintervals $A=[d, m], B=[m, 2 d]$ and $C=[2 d, u]$ are obtained, where $E[+]=[z, u]$. The map $x \rightarrow 2 x$ from $A$ into $B$ is both injective and onto: $(A, \cdot)$ is a max thread while $E[+]=B \cup C$ is a min thread under both operations. Moreover, $A E[+]=[m, d u]=B$, since $d+d=d u$ and $a+a=a u$ for each element $a$ in $A$.

In subcase $C S=E[+]=E[\cdot]$. Subintervals $A=[d, z], B=[z, m]$ and $C=[m, u]$ result, where $(A, \cdot),(A,+)$ and $(B, \cdot)$ are max threads, while $(C, \cdot),(C,+)$ and $(B,+)$ are min threads. From this $B C=\{m\}$ and $(A C) \cap B$ $=\{m\}$, resulting in $A C=[m, d u] \subset C$. Similarly $A+B=[d+m, z] \subset A$ and $A+C=[d+u, z] \subset A$, where $d+u=(d+u) \wedge(d+m)$ in $A$. In addition we specify that $C(A+B)=\{m\}$ and $a+c=a+a c, u(a+c)=a c$, for each $a \in A$ and $c \in C$. Both $(S,+)$ and $(S, \cdot)$ are examples of case III operations.

In subcase $\mathrm{D}$ addition is max. Let $A=[d, m], B=[m, u]$. Then for $a \in A$ and $b \in B$, we obtain $a b+m=m=a b$. Hence $A B=\{m\}$. This example is essentially that of subcase III-A.

Subcase E cannot occur unless $z=m$. With this property we obtain $E[+]=[d, z]$. Let $B=[z, u]$. Then $b u=b+b$ for each $b \in B$ and $E[+] A=[d u, z] \subset E[+]$. The addition is given by the case II operations, modified by the substitution of $d$ for $u$ and vice versa. In essence there is no difference between subcases $\mathrm{E}$ and $\mathrm{B}$ of the case III examples.

This material formed part of the author's dissertation, under the direction of Professor Michael Friedberg. I would like to thank Professor Dennison Brown for his suggestions and Raymond Houston for pointing out the result of Theorem 11.

\section{References}

[1] H. Anzai, 'On Compact Topological Rings', Proc. Imp. Acad. Tokyo 19 (1943), 513-615.

[2] A. H. Clifford and G. B. Preston, The Algebraic Theory of Semigroups, Vol. I (Amer. Math. Soc., 1961).

[3] M. Henriksen, 'The $a^{n(a)}=a$ Theorem for Semirings', Math. Japon. 5 (1958), 21-24.

[4] M. O. Poinsignon Grillet, 'Subdivision Rings of a Semiring', Fund. Math. 67 (1970), 67-74.

[5] R. J. Koch and A. D. Wallace, 'Notes on Inverse Semigroups', Rev. Roum. de Math. Pures et Appl. 9 (1964), 1-6.

[6] D. R. La Torre, The Radical of a Semiring, Master's Thesis, The University of Tennessee, Knoxville (1962).

[7] K. R. Pearson, 'Compact Semirings which are Multiplicatively Groups or Groups with Zero', Math. Zeitschr. 106 (1968), 388-394. 
[8] K. R. Pearson, 'The Three Kernels of a Compact Semiring', J. Austral. Math. Soc. 10(1969), 299-319.

[9] J. Selden, 'A Note on Compact Semirings', Proc. Amer. Math. Soc. 15 (1964), 882-886.

[10] A. D. Wallace, 'The Structure of Topological Semigroups', Bull. Amer. Math. Soc. 61 (1955), 95-112.

Department of Mathematics

University of Alberta

Edmonton

Canada 